(2) OPEN ACCESS

\title{
Reduced sexual contacts with non-steady partners and less PrEP use among MSM in Belgium during the first weeks of the COVID-19 lockdown: results of an online survey
}

\author{
Thiijs Reyniers @ , ${ }^{1}$ Anke Rotsaert, ${ }_{1}^{1}$ Estrelle Thunissen, ${ }^{2}$ Veerle Buffel, ${ }^{2}$ \\ Caroline Masquillier, ${ }^{2}$ Ella Van Landeghem, ${ }^{1}$ Jef Vanhamel, ${ }^{1}$ Christiana Nöstlinger, ${ }^{1}$ \\ Edwin Wouters, ${ }^{2}$ Marie Laga, ${ }^{1}$ Bea Vuylsteke ${ }^{1}$
}

${ }^{1}$ Department of Public Health, Institute of Tropical Medicine, Antwerpen, Belgium ${ }^{2}$ Department of Sociology, University of Antwerp, Antwerpen, Belgium

\section{Correspondence to} Dr Thijs Reyniers, Department of Public Health, Institute of Tropical Medicine, Antwerpen 2000, Belgium; treyniers@itg.be

Received 24 August 2020 Revised 5 October 2020 Accepted 17 October 2020 Published Online First 10 November 2020

\section{Check for updates}

(c) Author(s) (or their employer(s)) 2021. Re-use permitted under CC BY-NC. No commercial re-use. See rights and permissions. Published by BMJ.

To cite: Reyniers $\mathrm{T}$ Rotsaert A, Thunissen E, et al. Sex Transm Infect 2021:97:414-419.

\section{ABSTRACT}

Objectives To examine changes in the occurrence of physical sex with non-steady partners among men who have sex with men (MSM) in Belgium during the first weeks of the COVID-19 lockdown and associations with sociodemographic factors, sexual practices, drug, alcohol and pre-exposure prophylaxis (PrEP) use. A secondary objective was to explore changes in PrEP use and the need for PrEP follow-up.

Methods A cross-sectional online survey. The questionnaire was available in Dutch, French and English, between April 10 and 27 (2020), and disseminated via sexual health and lesbian, gay, bisexual, trans, queer or intersex organisations throughout Belgium. Eligibility criteria included being 18 years or older, not being exclusively heterosexual and living or being born in Belgium.

Results The sample included 694 MSM. Physical sex with non-steady partners decreased from $59.1 \%$ to $8.9 \%$ during the first weeks of the lockdown. Those who had sex with non-steady partners were significantly more likely to be HIV positive, to use PrEP or to have engaged in sexual practices such as group sex, chemsex and sex work before the lockdown, compared with their counterparts. Among those who used PrEP before the lockdown, 47.0\% stopped using PrEP, 19.7\% used event-driven PrEP and $33.3 \%$ used daily PrEP during the lockdown. Almost two-thirds of PrEP users had a PrEP care appointment in the weeks before the lockdown and a minority received follow-up elsewhere or online. Some PrEP users had concerns regarding their follow-up.

Conclusions MSM in our survey substantially reduced sexual contact with non-steady partners during the first weeks of the COVID-19 lockdown, suggesting that the risk for HIV and STI transmission in this period was low. We recommend ensuring access to sexual health services, such as HIV testing and follow-up for PrEP for the small group having multiple sex partners and engaging in sexual practices such as chemsex, or group sex, even in times of a pandemic threat.

\section{INTRODUCTION}

The current COVID-19 pandemic poses an unprecedented threat to public health. ${ }^{1}$ Preventive measures taken to control this pandemic have disrupted everyday life globally. Like other countries, the Belgian government imposed guidelines and measures restricting close physical contact to control COVID-19. ${ }^{2}$ In the first weeks of this initial 'lockdown', from 18 March to 10 May 2020, these measures included restricting real-life social contact to the household, while keeping a distance of 1.5 $\mathrm{m}$ from non-household members, also referred to as 'physical distancing'. Such physical distancing measures and stay-at-home recommendations are likely to impact on social life in general. In addition, these measures will have influenced sexual relations and frequency of physical sex between people who are not part of the same household, such as people living alone.

Having physical sex with partners outside the household while adhering to these physical distancing measures may be difficult, or even impossible. Men who have sex with men (MSM) are more likely to be non-monogamous, an inclusive term to indicate all ways in which MSM can have emotional and/or sexual bonds, such as being single with casual sex partners, serial monogamy, having multiple partners or open relationships. ${ }^{3-5}$ A subgroup of MSM has multiple casual sex partners, independent of being in a steady relationship and frequently engages in sexual behaviours that are shown to be associated with an elevated risk for HIV infection such as group sex or sex under the influence of recreational drugs. ${ }^{6-8}$ As a result, some MSM are at high risk for HIV and STIs, such as gonorrhoea and syphilis. ${ }^{9-11}$ It is hypothesised that preventive COVID-19 measures have an impact on sexual practices of MSM and that, consequently, their risk of acquiring HIV and STIs is reduced. ${ }^{12}$ However, evidence of reduced sexual contacts is currently scarce and may help to estimate the potential impact of the COVID-19 preventive measures on the STI and HIV epidemics.

Since June 2017, pre-exposure prophylaxis (PrEP) for HIV prevention is available and partly reimbursed for individuals at high risk for HIV infection in Belgium. ${ }^{13}$ PrEP users can opt for daily or event-driven PrEP and are required to visit an HIV centre every 3 months. We do not know to 
what extent PrEP users have reduced or stopped using PrEP in the first weeks of the COVID-19 lockdown period, because of reduced need resulting from decreased sexual activity. Moreover, during the first weeks of the COVID-19 lockdown, physical access to non-essential health services, such as PrEP care, was closed or substantially reduced. It remains unclear to what extent there was an unmet need for follow-up for PrEP in this period.

The objective of this study was to examine changes in the occurrence of physical sex with non-steady partners among MSM in Belgium in the first weeks of the COVID-19 lockdown period, and associations with sociodemographic factors, sexual practices associated with an elevated risk for HIV infection, drug, alcohol and PrEP use before the lockdown. A secondary objective was to explore changes in PrEP use and the need for follow-up for PrEP. The knowledge gained could help identify a subgroup of MSM who are more prone to taking health risks and for whom sexual health services would need to be ensured in times of a pandemic threat. The insights could help to reconsider the provision of PrEP during and after this lockdown period. Hence, the results can be important to anticipate next-wave preventive COVID-19 measures or novel pandemic threats that require physical distancing.

\section{METHODS}

In this cross-sectional survey, we collected self-reported data using an online questionnaire. The study was set up in collaboration with a Flemish sexual health organisation ('Sensoa') and a lesbian, gay, bisexual, trans, queer or intersex (LGBTQI) umbrella organisation ('Çavaria'). A link to the questionnaire was disseminated by sexual health and LGBTQI organisations throughout Belgium, via their social media accounts (eg, Facebook) and online newsletters.

The online questionnaire was available in Dutch, French and English, between April 10 and 27 (2020), that is, during the Belgian lockdown period starting on 18 March 2020, before any measure or restriction was loosened. Participants needed to be 18 years or older, not exclusively heterosexual and born in or living in Belgium.

Eligible participants provided consent by agreeing to participate, after having been informed about the study and its procedures. We developed the questionnaire based on previous European and Belgian studies among MSM and PrEP users. ${ }^{614-16}$ It was pilot tested within the research team and among members from community organisations. The questionnaire included questions on sociodemographic characteristics (see table 1), social contact, sexual practices associated with an elevated risk for HIV infection, drug and alcohol use,

Table 1 Associations between 'reported sex with non-steady partners since lockdown' and sociodemographic factors, sexual practices, drug, alcohol and PrEP use

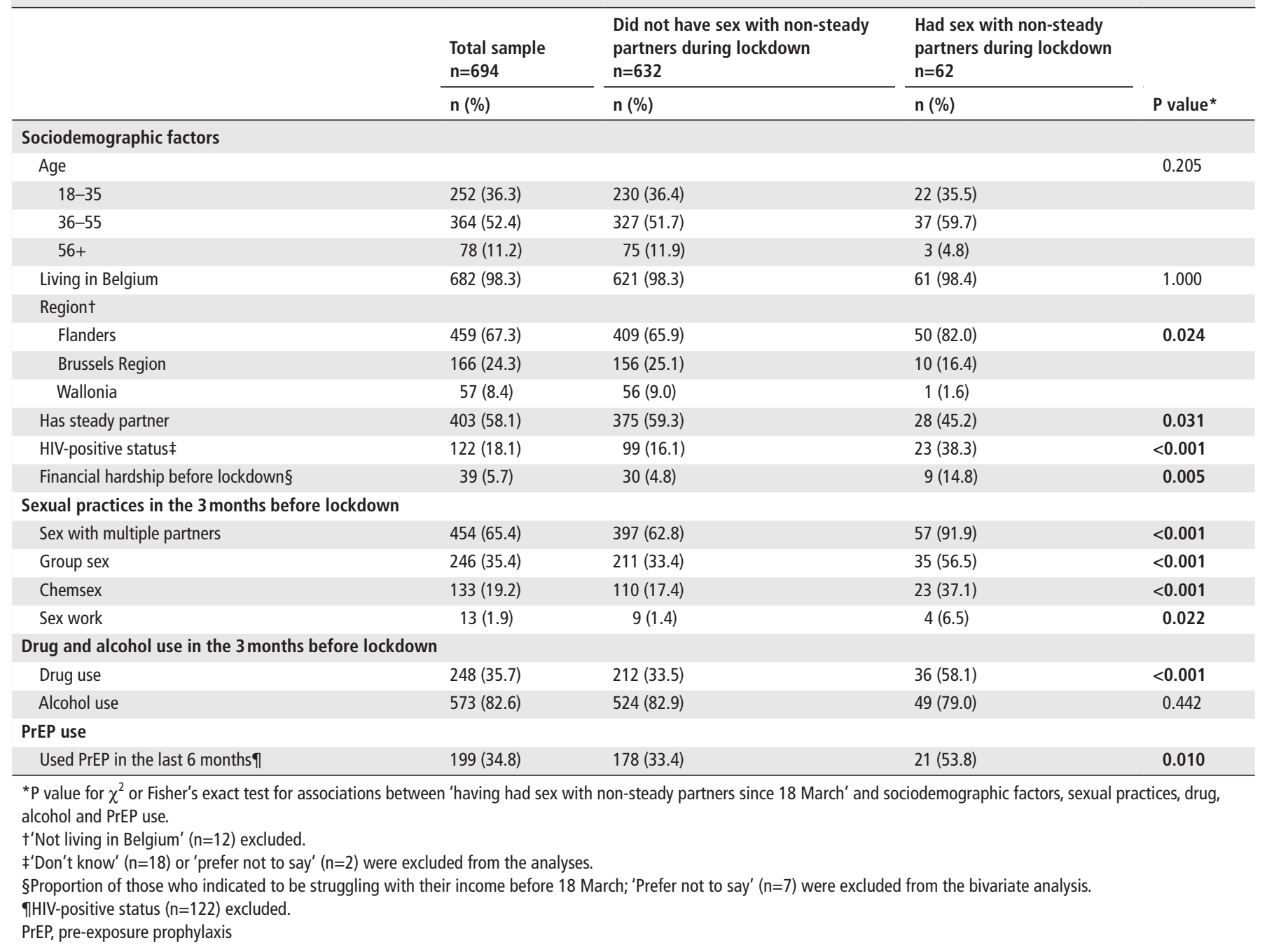




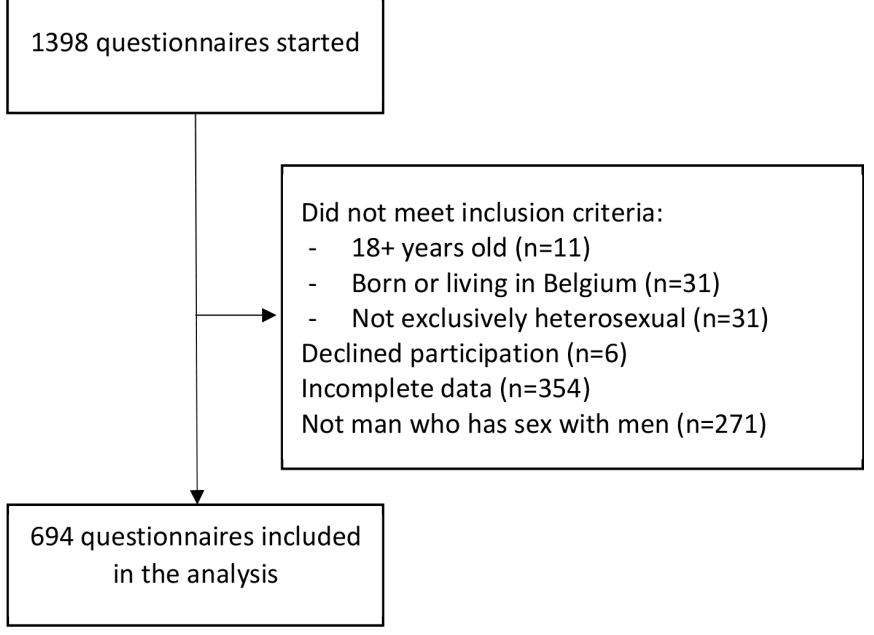

Figure 1 Sample selection.

depressive symptoms, anxiety and social support, PrEP use before and after 18 March 2020 and concerns towards PrEP use follow-up.

Sexual contact with non-steady partners was measured by asking whether close physical contact $(<1.5 \mathrm{~m})$ had occurred with casual (non-anonymous and non-steady) sex partners and with anonymous sex partners. We asked whether these had occurred before and after 18 March 2020, without specifying the length of the period. Financial hardship was measured by asking participants to what extent they felt they were struggling or living comfortably with their income before the COVID-19 lockdown in Belgium. Participants responded 'yes' or 'no' when asked whether they engaged in chemsex or slamming in the 3 months before 18 March.

For this analysis, we selected fully completed questionnaires from participants who indicated to be male, and to have sex with men. We excluded transwomen due to small group size $(n=7)$. We conducted a descriptive analysis using IBM SPSS Statistics V.25. A $\chi^{2}$ or Fisher's exact test was used to examine associations between sociodemographic factors, risk behaviour and PrEP use, and having close physical contact with non-steady (casual or anonymous) sex partners during the early phase of the COVID-19 lockdown (ie, the outcome).

\section{RESULTS}

We included 694 questionnaires in the analysis (figure 1). The median time needed to complete the questionnaire was about $12 \mathrm{~min}$. About half of the sample (52.4\%) was between 36 and 55 years old. Almost all participants were currently living in Belgium (98.3\%), the majority of those in Flanders region $(67.3 \%)$. About 18.1\% indicated to be HIV positive and 199 (34.8\%) participants reported to have used PrEP in the six preceding months. About $82.6 \%$ had used alcohol in the 3 months before the lockdown, and $21.3 \%$ indicated to be drinking more alcohol since the lockdown.

\section{Changes in the occurrence of physical sex with non-steady partners}

The proportion of participants reporting physical sex with casual partners decreased from $52.7 \%$ to $7.1 \%$ during the first weeks of the lockdown, and with anonymous sex partners from $47.4 \%$ to $3.9 \%$ (figure 2). Sixty-two participants (8.9\%) reported physical sex with non-steady partners (casual or anonymous) since 18 March 2020.

Participants who had sex with non-steady partners during the lockdown were significantly more likely to be single and to be struggling financially with their income, when compared with those who did not have sex with non-steady partners during the lockdown (table 1). Among those who had sex with non-steady partners during the lockdown, about $71.0 \%$ were either HIV positive $(n=23)$ or taking PrEP $(n=21)$ in the 6 months before. Participants who had sex with non-steady partners during the lockdown were more likely to have had multiple sex partners, engaged in group sex, chemsex and sex work and to have used drugs in the 3 months before the lockdown, when compared with their counterparts.

\section{Changes in PrEP use and follow-up}

One participant had stopped taking PrEP before the lockdown. Among those who were using either daily or event-driven PrEP, about half $(47.0 \%)$ had stopped PrEP use during the lockdown (table 2). Of those who used event-driven PrEP before

\section{Sex with non-steady sex partners}

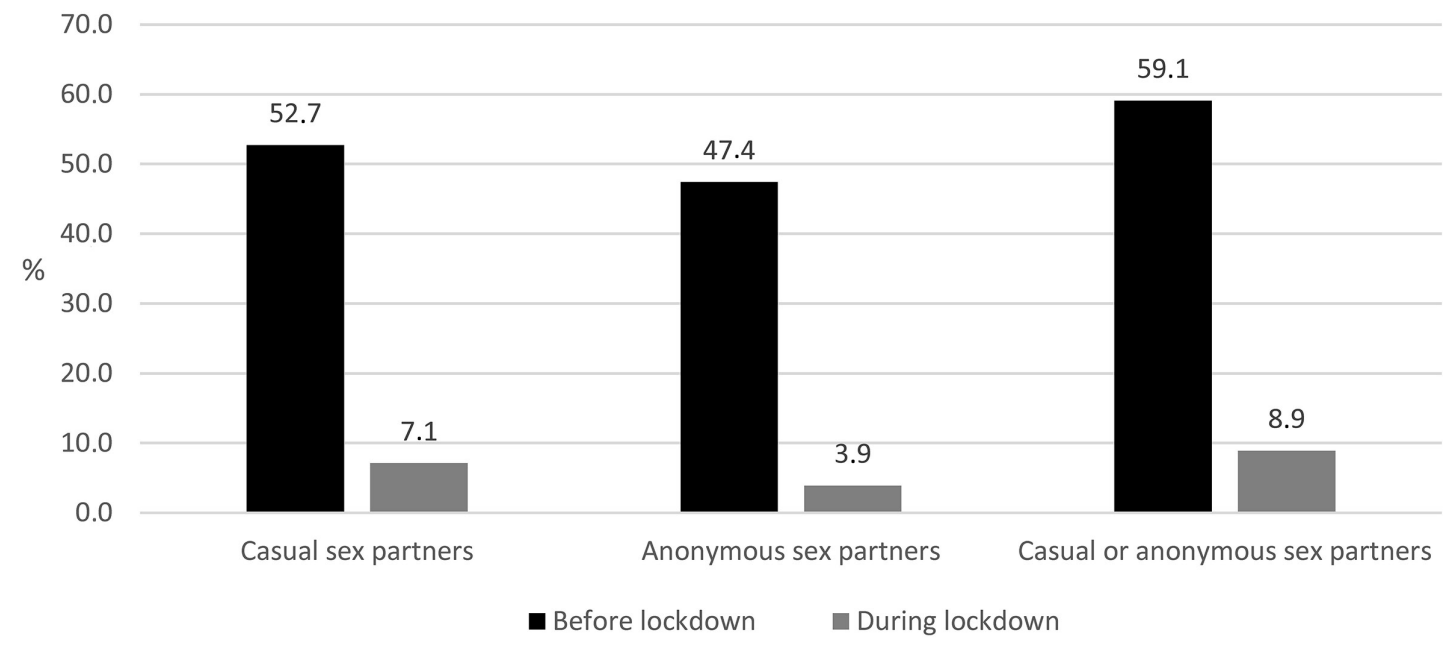

non-steady sex partners with whom physical sex had occurred

Figure 2 Proportion of participants reporting sex with non-steady sex partners, before and during first weeks of the COVID-19 lockdown. 
Table 2 PrEP use before and during early lockdown, that is, since 18 March 2020 ( $\mathrm{N}=198)^{*}$

\begin{tabular}{|c|c|c|c|c|}
\hline \multicolumn{2}{|c|}{ PrEP use before lockdown* } & \multicolumn{3}{|c|}{ PrEP use during early lockdown } \\
\hline & Total & None & Event-driven & Daily \\
\hline & n (\%) & $\begin{array}{l}n \text { ( } \% \text { of } \\
\text { 'before') }\end{array}$ & $\begin{array}{l}\text { n (\% of } \\
\text { 'before') }\end{array}$ & $\begin{array}{l}n(\% \text { of } \\
\text { 'before') }\end{array}$ \\
\hline Event-driven & $90(45.5)$ & $51(56.7)$ & $35(38.9)$ & $4(4.4)$ \\
\hline Daily & $108(54.5)$ & $42(38.9)$ & $4(3.7)$ & $62(57.4)$ \\
\hline Total & $198(100)$ & $93(47.0)$ & $39(19.7)$ & $66(33.3)$ \\
\hline
\end{tabular}

*One participant reported to have stopped using PrEP before the lockdown and is excluded from the analysis.

PrEP, pre-exposure prophylaxis

the lockdown, the majority had stopped using PrEP (56.7\%) or continued using event-driven PrEP (38.9\%).

Among those who used PrEP daily before the lockdown, about $38.9 \%$ had stopped using PrEP and the majority (57.4\%) had continued using daily PrEP. One participant reported to have restarted daily use after reading that he believed it could help against COVID-19. Four participants switched from daily to event-driven PrEP and four switched from event-driven to daily PrEP.

About $15.4 \%$ of those using event-driven PrEP during the lockdown $(n=6)$ reported sex with non-steady partners during the lockdown, while this was $16.7 \%$ among daily users $(n=11)$ and $4.3 \%$ among those who reported to have stopped using $\operatorname{PrEP}(\mathrm{n}=4)$.

Almost two-thirds of the PrEP users (64.9\%) reported that their last PrEP care appointment took place less than 3 months (90 days or sooner) before completion of the questionnaire. One hundred and twenty PrEP users (60.3\%) indicated no changes regarding their follow-up. Forty-five (22.6\%) had their PrEP care appointment postponed, 8 $(4.0 \%)$ were followed up online and $4(2.0 \%)$ were followed up elsewhere (eg, family physician). Three PrEP users (1.5\%) reported not being followed up (eg, buying PrEP online). Sixteen PrEP users (8.0\%) worried that they would not have sufficient PrEP pills and $22(11.1 \%)$ received prescriptions online. Six PrEP users (3.0\%) reported being concerned about not having been tested or about the time between the previous and the next HIV or STI test.

\section{DISCUSSION}

MSM in our survey reduced sexual contact with non-steady partners from $59.1 \%$ to $8.9 \%$ during the first weeks of the COVID-19 lockdown. Those having sex with non-steady partners during the early phase of the lockdown were significantly more likely to be struggling financially and to have engaged in sexual practices with an elevated risk for HIV infection, such as group sex, chemsex and sex work before the lockdown. About half of those who used PrEP before the lockdown (47.0\%) had stopped using PrEP, 19.7\% used event-driven PrEP and 33.3\% daily PrEP during the lockdown.

An important finding is the substantial reduction of sex with non-steady partners during the first weeks of the lockdown period among the MSM in our sample. This is in line with studies in the USA and Australia where MSM indicated fewer sexual partners or having less opportunities for having sex. ${ }^{17-19}$ The reduced sexual activities with sexual partners outside the household is likely to result in reduced HIV and STI transmission among MSM. Among those who had sex with non-steady partners during the lockdown in our study, more than two-third were using PrEP or were HIV positive. In Belgium, about 97\% of people living with HIV are in follow-up for treatment and $97 \%$ of those are virally suppressed (HIV viral load of less than 200 copies $/ \mathrm{mL}) .{ }^{20}$ Hence, these findings suggest that the risk for HIV transmission was low during this phase of the COVID-19 lockdown in Belgium. Whether and to what extent STI and HIV infections actually decreased during the COVID-19 period is yet to be examined.

Among the MSM using PrEP before the lockdown period, 47.0\% had stopped using PrEP, which is in line with a study among MSM in Australia. ${ }^{19}$ While it cannot be excluded that they stopped using PrEP to ensure sufficient pill supplies, only $8.0 \%$ in our study reported being concerned about not having sufficient pills. Moreover, considering that almost two-thirds had been on a PrEP care appointment within 90 days before completion of the survey and that only $22.6 \%$ had their appointment postponed, it is unlikely that they stopped using PrEP due to not having access to PrEP care. These findings suggest that they stopped using PrEP due to perceiving a reduced risk for HIV infection.

PrEP is only to be taken in periods of high risk and does not need to be taken in other times. ${ }^{21}$ Therefore, it could be anticipated that more PrEP users would switch from daily to event-driven PrEP, providing them the opportunity to reduce pill use while keeping the possibility of using PrEP in case of a sexual encounter. Only five participants switched from daily PrEP to event-driven. Moreover, the majority of those using daily before the lockdown (57.4\%) remained to use PrEP daily during the lockdown, in line with an Australian study. ${ }^{19}$ Potential reasons for continuing daily PrEP use could be that such daily users prefer to keep their routine or that they do not exclude the possibility of unplanned sexual encounters. Another reason may be the lack of awareness, available knowledge or support on how to stop and restart PrEP use. Therefore, we recommend that clear guidelines should be available, preferably online, to support PrEP users in safely stopping, starting and restarting with PrEP use. Ideally, such guidelines could also include recommendations on when and how to test for HIV before restarting with PrEP use.

Many PrEP users either had a PrEP care appointment in the 3 months before the lockdown, or received follow-up elsewhere (eg, family physician) or online (eg, online prescriptions). These findings suggest that the need for follow-up was low at the time of data collection and that follow-up services were already well adapted to this novel situation. However, our study was undertaken in the first weeks, in which preventive measures for COVID-19 impacted on everyday life in Belgium. The need for follow-up may be higher when COVID-19 restrictions are loosened, and sexual activities among non-steady partners may increase again among MSM.

Only a minority $(8.9 \%)$ of our sample had sex with nonsteady partners, suggesting that the majority adhered well to the COVID-19 regulations when it comes to close physical contact with sex partners outside the household. About $8.0 \%$ of the PrEP users were concerned about insufficient pill supply, and $3.0 \%$ were concerned about not being tested for HIV or STIs. These findings suggest that a minimum of sexual health services, such as HIV testing, needs to be ensured for a subgroup of MSM, even in times of a pandemic threat during which sex with partners outside the household is prohibited. The unique situation of reduced physical contact and a societal lockdown may be a good opportunity to consider alternative forms of PrEP follow-up and sexual health services, such as online counselling or home-based sampling. ${ }^{22}$ Additionally, we suggest further research is needed 
to investigate what minimum package for PrEP follow-up would be feasible and for whom.

Those who had sex with non-steady partners during the lockdown in our sample were more likely to have engaged in group sex, chemsex or sex work before the lockdown. Such sexual practices are shown to be associated with condomless sex and an elevated risk for HIV infection. ${ }^{23-27}$ Additionally, we found that participants struggling financially were more likely to have had sex with non-steady partners during the lockdown. Financial hardship among MSM is shown to be associated with a higher likelihood of participating in sexual risky situations (eg, alcohol or drug use before or during sex), condomless anal intercourse, transactional sex and drug use. ${ }^{28-30}$ These findings confirm that among MSM, a subgroup can be identified that is vulnerable for acquiring HIV or other STIs. We recommend HIV and STI prevention organisations to provide guidance on how to reduce the risk for HIV infections and STIs when engaging in sexual practices such as group sex, chemsex or sex work. Additionally, we recommend tailored messages and information for reducing the risk of COVID-19 while having sex with non-steady partners, engaging in chemsex, group sex or doing sex work, in particular during a next societal lockdown or when preventive measures are taken to reduce close physical social contact. Home-based sampling and online follow-up may be valid alternatives to test those who may have acquired HIV or STIs before or during a lockdown period. We recommend prioritising this subgroup vulnerable for acquiring HIV and STIs when restarting on-site sexual health services.

The use of an online questionnaire limits the generalisability of the findings due to the inherent self-selection bias. Persons associated with LGBTQI or sexual health organisations were more likely to receive a link to the questionnaire. The MSM in our sample were less likely to be struggling financially $(5.7 \%)$, more likely to have a steady partner $(58.1 \%)$ and more likely to be HIV positive (18.1\%), when compared with a survey among European MSM (16.9\%, $38.6 \%$ and $10.0 \%$, respectively). ${ }^{6}$ Data collected later in the lockdown period could have resulted in higher proportions of participants having sex with non-steady partners, or in more or fewer PrEP users being concerned about their follow-up. We have not measured the use of condoms, which could have provided additional insights into the potential transmission of HIV or STIs during the COVID-19 period. Recall bias concerning sexual behaviour in the 3 months before COVID-19 cannot be excluded. The lack of longitudinal data prevents us from examining the impact of implementing additional or loosening of physical distancing restrictions on the sexual behaviour of MSM. In countries with less stringent preventive measures and restrictions to control the COVID-19 epidemic, MSM may not have reduced sexual activities with non-steady partners and PrEP use to the same extent.

\section{CONCLUSIONS}

MSM in our survey substantially reduced sexual contact with non-steady partners during the first weeks of the COVID-19 lockdown, suggesting that the risk for HIV and STI transmission in this period was low. Our findings indicate that the need for PrEP care appointments was low and that follow-up services were already well adapted to this novel situation. We recommend ensuring access to sexual health services, such as HIV testing and PrEP follow-up, for a subgroup of MSM who may be vulnerable for acquiring HIV or STIs.

Handling editor Jo Gibbs

\section{Key messages}

- Men who have sex with men (MSM) substantially reduced sexual contact with non-steady partners during the first weeks of COVID-19 lockdown, suggesting the risk for HIV and STI transmission was low.

- About half of all pre-exposure prophylaxis (PrEP) users have stopped using PrEP during the first weeks of COVID-19 lockdown, and the need for PrEP follow-up was low.

- We recommend ensuring access to sexual health services, such as PrEP follow-up, for a subgroup of MSM who may be vulnerable for acquiring HIV or STIs.

Acknowledgements We would like to thank all study participants for completing the online questionnaire. We would like to thank Sensoa, Çavaria and all other lesbian, gay, bisexual, trans, queer or intersex (LGBTQI) and sexual health organisations for their help in collecting the data.

Contributors All authors were involved in setting up the study and data collection. TR and AR conducted the analysis. TR drafted the manuscript, and all authors commented on subsequent versions. All authors approved the final manuscript.

Funding TR is a postdoctoral fellow of the Research Foundation - Flanders. The study consortium received funding from the Research Foundation - Flanders as an SBO-project (S004919N).

\section{Competing interests None declared.}

\section{Patient consent for publication Not required.}

Ethics approval The study received ethical approval of the Institutional Review Board of the Institute of Tropical Medicine, Antwerp (IRB 1378/20).

Provenance and peer review Not commissioned; externally peer reviewed. Data availability statement Data are not available due to ethical restrictions.

Open access This is an open access article distributed in accordance with the Creative Commons Attribution Non Commercial (CC BY-NC 4.0) license, which permits others to distribute, remix, adapt, build upon this work non-commercially, and license their derivative works on different terms, provided the original work is properly cited, appropriate credit is given, any changes made indicated, and the use is non-commercial. See: http://creativecommons.org/licenses/by-nc/4.0/.

\section{ORCID iD}

Thijs Reyniers http://orcid.org/0000-0003-3756-921X

\section{REFERENCES}

1 European Centre for Disease Prevention and Control. Resurgence of reported cases of COVID-19 in the EU/EAA, the UK and EU candidate and potential candidate countries, 2020.

2 Belgian Federal Government. Coronavirus: reinforced measures by the Belgian Federal Government, 2020. Available: https://www.belgium.be/en/news/2020/coronavirus_ reinforced_measures [Accessed 11 Aug 2020].

3 van Eeden-Moorefield B, Malloy K, Benson K. Gay Men's (Non)Monogamy Ideals and Lived Experience. Sex Roles 2016;75:43-55.

4 Newcomb ME, Ryan DT, Garofalo R, et al. The effects of sexual partnership and relationship characteristics on three sexual risk variables in young men who have sex with men. Arch Sex Behav 2014;43:61-72.

5 Philpot SP, Bavinton BR, Prestage G, et al. Exploring diversity in HIV research in the sexual partnerships of Australian gay and bisexual men. Arch Sex Behav 2020;49:2069-80.

6 European Centre for Disease Prevention and Control. EMIS 2017: The European MenWho-Have- Sex-With-Men Internet Survey - key findings from 50 countries, 2019

7 Reyniers T, Nöstlinger C, Laga M, et al. Choosing between daily and Event-Driven pre-exposure prophylaxis. J Acquir Immune Defic Syndr 2018;79:186-94.

8 McCall H, Adams N, Mason D, et al. What is chemsex and why does it matter? BMJ 2015;351:h5790.

9 European Centre for Disease Prevention and Control. HIV and men who have sex with men - monitoring implementation of the Dublin Declaration on partnership to fight HIVIAIDS in Europe and Central Asia: 2018 progress report, 2020.

10 European Centre for Disease Prevention and Control. Gonorrhoea - annual epidemiological report for 2018, 2018.

11 European Centre for Disease Prevention and Control. Syphilis - annual epidemiological report for 2018, 2018.

12 Alpalhão M, Filipe P. The impacts of isolation measures against SARS-CoV-2 infection on sexual health. AIDS Behav 2020:1-2. 
13 Vuylsteke B, Reyniers T, Lucet C, et al. High uptake of pre-exposure prophylaxis (PrEP) during early roll-out in Belgium: results from surveillance reports. Sex Health 2019;16:80-3.

14 Reyniers T, Nöstlinger C, Laga M, et al. Choosing between daily and event-driven pre-exposure prophylaxis: results of a Belgian PrEP demonstration project. J Acquir Immune Defic Syndr 2018;79:186-94.

15 Vuylsteke B, Reyniers T, De Baetselier I, et al. Daily and event-driven pre-exposure prophylaxis for men who have sex with men in Belgium: results of a prospective cohort measuring adherence, sexual behaviour and STI incidence. J Int AIDS SOC 2019;22:e25407.

16 Bullinger J, Reyniers T, Vuylsteke B, et al. Congruence between hypothetical willingness to use pre-exposure prophylaxis (PreP) and eligibility: an online survey among Belgian men having sex with men. Int J Environ Res Public Health 2019;16. doi:10.3390/ ijerph16224411. [Epub ahead of print: 11 Nov 2019].

17 Sanchez TH, Zlotorzynska M, Rai M, et al. Characterizing the impact of COVID-19 on men who have sex with men across the United States in April, 2020. AIDS Behav 2020;24:2024-32.

18 Hammoud MA, Maher L, Holt M, et al. Physical distancing due to COVID-19 disrupts sexual behaviors among gay and bisexual men in Australia: implications for trends in HIV and other sexually transmissible infections. J Acquir Immune Defic Syndr 2020;85:309-15.

19 Chow EPF, Hocking JS, Ong JJ, et al. Changing the use of HIV pre-exposure prophylaxis among men who have sex with men during the COVID-19 pandemic in Melbourne, Australia. Open Forum Infect Dis 2020;7:ofaa275.

20 Sciensano. Epidemiologie van AIDS en HIV-infectie in België toestand op 31 december 2018, 2019.
21 Haberer JE, Bangsberg DR, Baeten JM, et al. Defining success with HIV preexposure prophylaxis: a prevention-effective adherence paradigm. AIDS 2015;29:1277-85.

22 Vanhamel J, Rotsaert A, Reyniers T, et al. The current landscape of pre-exposure prophylaxis service delivery models for HIV prevention: a scoping review. BMC Health Serv Res 2020;20:704.

23 Prestage GP, Hudson J, Down I, et al. Gay men who engage in group sex are at increased risk of HIV infection and onward transmission. AIDS Behav 2009;13:724-30.

24 Mimiaga MJ, Reisner SL, Bland SE, et al. Sex parties among urban MSM: an emerging culture and HIV risk environment. AIDS Behav 2011;15:305-18.

25 Vuylsteke B, Semde G, Sika L, et al. High prevalence of HIV and sexually transmitted infections among male sex workers in Abidjan, Cote d'Ivoire: need for services tailored to their needs. Sex Transm Infect 2012;88:288-93.

26 Baral SD, Friedman MR, Geibel S, et al. Male sex workers: practices, contexts, and vulnerabilities for HIV acquisition and transmission. Lancet 2015;385:260-73.

27 Colfax GN, Mansergh G, Guzman R, et al. Drug use and sexual risk behavior among gay and bisexual men who attend circuit parties: a venue-based comparison. J Acquir Immune Defic Syndr 2001;28:373-9.

28 Ayala G, Bingham T, Kim J, et al. Modeling the impact of social discrimination and financial hardship on the sexual risk of HIV among Latino and black men who have sex with men. Am J Public Health 2012;102(Suppl 2):S242-9.

29 Duncan DT, Park SH, Schneider JA, et al. Financial hardship, Condomless ana intercourse and HIV risk among men who have sex with men. AIDS Behav 2017:21:3478-85.

30 Park SH, Al-Ajlouni Y, Palamar JJ, et al. Financial hardship and drug use among men who have sex with men. Subst Abuse Treat Prev Policy 2018;13:19. 